\title{
ANALOGUES OF THE BRAID GROUP WHOSE GRAPHS ARE STARS
}

\author{
by ANDREW K. NAPTHINE \\ (Received 14th December, 1983)
}

\section{Introduction}

Let $G$ be a group having presentation

$$
\left\langle\left\{x_{i}: i=1, \ldots, n+1\right\} \mid r_{i j}, 1 \leqq i<j \leqq n+1\right\rangle
$$

where

$$
r_{i j}=x_{i} x_{j} x_{i} x_{j}^{-1} x_{i}^{-1} x_{j}^{-1}:=\left(x_{i}, x_{j}\right)
$$

or

$$
x_{i} x_{j} x_{i}^{-1} x_{j}^{-1}:=\left[x_{i}, x_{j}\right]
$$

a braid relator and a commutator respectively, and define the graph of such a group as having vertices labelled $x_{1}, \ldots, x_{n+1}$ and such that $x_{i}$ and $x_{j}$ are joined if and only if $r_{i j}=\left(x_{i}, x_{j}\right)$.

With this definition Artin's braid group, $B_{n}$, [2] has graph which is an interval and in [1] Al-bar and Johnson examine those groups whose graphs are regular of degree two.

In this paper we investigate those groups, henceforth called $G_{n}$, whose graph has exactly one vertex of degree $n$ and all others have degree one, in other words the bipartite graphs $K_{1, n}$. (For graph theoretic terminology and notation see Wilson [6]).

\section{The derived group of $G_{n}$}

We shall always assume that $x_{1}$ is a vertex of degree one and that the vertex of degree $n$ is $x_{2}$. Note that any necessary re-labelling of vertices corresponds to elementary Nielsen transformations and therefore preserves the group (up to isomorphism).

Using an argument similar to that of Gorin and Lin [3] it can be seen that $G_{n} / G_{n}^{\prime} \cong Z$, and we may choose $\left\{x_{1}^{i}: i \in Z\right\}$ as a Schreier transversal for $G_{n}^{\prime}$. The Reidemeister-Schreier rewriting process now yields generators

$$
X_{j, i}:=x_{1}^{i} x_{j} x_{1}^{-(i+1)}, \quad j=2, \ldots, n+\hat{1,}
$$


and relations

$$
\begin{array}{rlr}
X_{2, i} X_{2, i+2} & =X_{2, i+1}, & \\
X_{j, i} & =X_{j, i+1}, & \\
X_{2, i} X_{j, i+1} X_{2, i+2}=X_{j, i} X_{2, i+1} X_{j, i+2}, \ldots, n+1 \\
X_{j, i} X_{k, i+1}=X_{k, i} X_{j, i+1}, & j=3, \ldots, n+1
\end{array}
$$

for all $i \in Z$.

It has been shown (in [5]) that this presentation can be reduced to a finite one, and this may be further modified (by Tietze transformations) to the following:

$$
\begin{gathered}
\left\langle u, v,\left\{a_{i}, \alpha_{i}: i=3, \ldots, n+1\right\}\right|\left[a_{i}, a_{j}\right],\left[\alpha_{i}, \alpha_{j}\right], \\
u a_{i} u^{-1}=\alpha_{i}, \\
u \alpha_{i} u^{-1}=\alpha_{i}^{2} a_{i}^{-1} \alpha_{i}, \\
v a_{i} v^{-1}=a_{i}^{-1} \alpha_{i}, \\
v \alpha_{i} v^{-1}=\left(a_{i}^{-1} \alpha_{i}\right)^{3} a_{i}^{-2} \alpha_{i},
\end{gathered}
$$

$$
\text { for } i, j \in\{3, \ldots, n+3\}>
$$

where $u=x_{2} x_{1}^{-1}, v=x_{1} x_{2} x_{1}^{-2}, a_{i}=x_{i} x_{1}^{-1}, \alpha_{i}=x_{2} x_{i} x_{1}^{-1} x_{2}^{-1}$. Note here, that when $n=2$ we have $B_{4}$ and the above presentation is that found by Gorin and Lin [3].

Write $T_{n}:=\left\langle\left\{a_{i}, \alpha_{i}: i=3, \ldots, n+1\right\}\right\rangle$, then the presentation (2) together with the relations $u^{-1} a_{i} u=a_{i} \alpha_{i}^{-1} a_{i}^{2}, u^{-1} \alpha_{i} u=a_{i}, v^{-1} a_{i} v=a_{i} \alpha_{i}^{-1} a_{i}^{3}, v^{-1} \alpha_{i} v=a_{i} \alpha_{i}^{-1} a_{i}^{4}$ for all $i=3, \ldots, n+1$, shows that $T_{n} \triangleleft G_{n}^{\prime}$. $G_{n}^{\prime} / T_{n}$ is $\langle u, v \mid\rangle$, a free group of rank two and we determine a presentation for $T_{n}$ as follows.

Define

$$
R_{n}=\left\langle\left\{b_{i}, \beta_{i}: i=3, \ldots, n+1\right\}\right|\left[b_{i}, b_{j}\right],\left[\beta_{i}, \beta_{j}\right]
$$

for $3 \leqq i<j \leqq n+1\rangle$,

a free product of two copies of $Z^{\times(n-1)}$ and let $U=\langle\mu, v \mid\rangle$ be free of rank two.

Consider the split extension $E$ of $R_{n}$ by $U$ having presentation

$$
\begin{aligned}
\left\langle\mu, v,\left\{b_{i}, \beta_{i}: i=3, \ldots, n+1\right\}\right|\left[b_{i}, b_{j}\right],\left[\beta_{i}, \beta_{j}\right] & \\
\mu b_{i} \mu^{-1} & =\beta_{i}, \\
\mu \beta_{i} \mu^{-1} & =\beta_{i}^{2} b_{i}^{-1} \beta_{i},
\end{aligned}
$$




$$
\begin{aligned}
& v b_{i} v^{-1}=b_{i}^{-1} \beta_{i}, \\
& v \beta_{i} v^{-1}=\left(b_{i}^{-1} \beta_{i}\right)^{3} b_{i}^{-2} \beta_{i},
\end{aligned}
$$

for $i, j \in\{3, \ldots, n+1\}\rangle$.

This gives us two extensions

$$
\begin{aligned}
& 1 \rightarrow T_{n} \rightarrow G_{n}^{\prime} \rightarrow G_{n}^{\prime} / T_{n} \rightarrow 1
\end{aligned}
$$

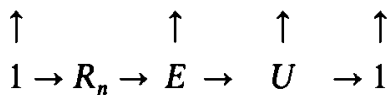

where all of the vertical arrows indicate isomorphisms and the square commutes. A modified version of the five lemma now implies that $R_{n}$ is isomorphic to $T_{n}$ so that $G_{n}^{\prime}$ is the split extension of a free product of two copies of $Z^{\times(n-1)}$ by a free group of rank two.

\section{The main theorem}

The structure of $G_{n}$ may be revealed by showing that $T_{n}$ is, in fact, normal in $G_{n}$ and not just in $G_{n}^{\prime}$. This may be done by direct calculation for

$$
\begin{array}{ll}
x_{1} a_{i} x_{1}^{-1}=a_{i}, & x_{1} \alpha_{i} x_{1}^{-1}=a_{i}^{-1} \alpha_{i}, \\
x_{2} a_{i} x_{2}^{-1}=\alpha_{i}, & x_{2} \alpha_{i} x_{2}^{-1}=\alpha_{i} a_{i}^{-1} \alpha_{i}, \\
x_{i} a_{i} x_{i}^{-1}=a_{i}, & x_{i} \alpha_{i} x_{i}^{-1}=\alpha_{i} a_{i}^{-1}, \\
x_{j} a_{i} x_{j}^{-1}=a_{i}, & x_{j} \alpha_{i} x_{j}^{-1}=a_{j} a_{i}^{-1} \alpha_{i} a_{j}^{-1},
\end{array}
$$

and

$$
\begin{array}{ll}
x_{1}^{-1} a_{i} x_{1}=a_{i}, & x_{1}^{-1} \alpha_{i} x_{1}=a_{i} \alpha_{i}, \\
x_{2}^{-1} a_{i} x_{2}=a_{i}^{-1} \alpha_{i} a_{i}^{-1}, & x_{2}^{-1} \alpha_{i} x_{2}=a_{i}, \\
x_{i}^{-1} a_{i} x_{i}=a_{i}, & x_{i}^{-1} \alpha_{i} x_{i}=\alpha_{i} a_{i}, \\
x_{j}^{-1} a_{i} x_{j}=a_{i}, & x_{i}^{-1} \alpha_{i} x_{j}=a_{i} a_{j}^{-1} \alpha_{i} a_{j},
\end{array}
$$

for $i, j \in\{3, \ldots, n+1\}$ with $i \neq j$.

Thus $T_{n}$ is normal in $G_{n}$ and, by putting each $a_{i}$ and $\alpha_{i}$ equal to the identity in $G_{n}$, that is putting $x_{1}=x_{i}$ for each $3 \leqq i \leqq n+1$, we obtain that $G_{n} / T_{n} \cong B_{3}$. In fact we prove the stronger result below. 
Theorem. (a) Let

$$
H_{0}=G_{n}^{\prime}, H_{1}=\left[H_{0}, H_{0}\right], \ldots, H_{k+1}=\left[H_{k}, H_{0}\right], \ldots
$$

be the lower central series of the group $G_{n}^{\prime}$. Then $T_{n}=\bigcap_{k=0}^{\infty} H_{k}$. In particular, $T_{n}$ is a fully invariant subgroup of both $G_{n}^{\prime}$ and $G_{n}$.

(b) The kernel of every homomorphism $\phi: G_{n} \rightarrow B_{3}$ contains $T_{n}$.

(c) The kernel of every epimorphism $\phi: G_{n} \rightarrow B_{3}$ coincides with $T_{n}$.

Proof. (a) Since $H_{0}=G_{n}^{\prime}, T_{n} \subseteq H_{0}$ and we proceed by induction.

Assume that for some $k \geqq 0, T_{n} \subseteq H_{k}$. By the relations (i)

$$
\alpha_{i}=u a_{i} u^{-1}=a_{i} a_{i}^{-1} u a_{i} u^{-1}=a q, \text { say },
$$

where

$$
q=a_{i}^{-1} u a_{i} u^{-1} \in\left[T_{n}, H_{0}\right] \subseteq\left[H_{k}, H_{0}\right]=H_{k+1}, \text { for } i=3, \ldots, n+1 .
$$

Thus $a_{i}^{-1} \alpha_{i}=q \in H_{k+1}$, for $i=3, \ldots, n+1$. Now from (iii) we have

$$
\begin{aligned}
& a_{i}=v^{-1}\left(a_{i}^{-1} \alpha_{i}\right) v \in H_{k+1}, \text { and therefore } \\
& \alpha_{i}=a_{i} q \in H_{k+1}, \text { for } i=3, \ldots, n+1 .
\end{aligned}
$$

Thus $T_{n} \subseteq H_{k+1}$, and, by induction, we have $T_{n} \subseteq \bigcap_{k=0}^{\infty} H_{k}$.

For the reverse inclusion, note that the image of the subgroup $\bigcap_{k=0}^{\infty} H_{k} \subseteq G_{n}^{\prime}$ under the natural projection $\tau: G_{n}^{\prime} \rightarrow G_{n}^{\prime} / T_{n}$ is contained in the intersection of the lower central series of $G_{n}^{\prime} / T_{n}$. However $G_{n}^{\prime} / T_{n}$ is free of rank two and a theorem of Magnus (see [4] p. 38) implies that this intersection is trivial. Thus $\bigcap_{k=0}^{\infty} H_{k} \subseteq \operatorname{ker} \tau=T_{n}$.

(b) Let $\phi: G_{n} \rightarrow B_{3}$ be an arbitrary homomorphism. Since $T_{n} \subseteq G_{n}^{\prime}$ we have $\phi\left(T_{n}\right) \subseteq \phi\left(G_{n}^{\prime}\right) \subseteq B_{3}^{\prime}$. From part (a) we may deduce that $\phi\left(T_{n}\right)$ is contained in the intersection of the lower central series of $B_{3}^{\prime}$. But $B_{3}^{\prime}$ is free, and again, by Magnus, this intersection is trivial and $\phi(T)=\{e\}$.

(c) Let $S=\operatorname{ker} \phi$. Then the homomorphism $G_{n} / G_{n}^{\prime} \rightarrow B_{3} / B_{3}^{\prime}$ induced by $\phi$ has kernel $S G_{n}^{\prime} / G_{n}^{\prime}$. Now finitely generated free groups have the Hopf property (see [4] p. 59) and since $G_{n} / G_{n}^{\prime}$ and $B_{3} / B_{3}^{\prime}$ are both infinite cyclic $S G_{n} / G_{n}^{\prime}$ is trivial so that $S \subseteq G_{n}^{\prime}$.

Furthermore, the restriction of $\phi$ to $G_{n}^{\prime}$ is onto $B_{3}^{\prime}$ which yields $G_{n}^{\prime} / S \cong B_{3}^{\prime}$, but $G_{n}^{\prime} / T_{n} \cong B_{3}^{\prime}$, both free groups of rank two and by part (b) $G_{n}^{\prime} / S$ is (isomorphic to) a factor group of $G_{n}^{\prime} / T_{n}$. Using the Hopf property again gives us that $S \subseteq T_{n}$ as required.

It should be noted here that the proof of this theorem is a straightforward generalization of that which may be found in Gorin and Lin [3].

\section{REFERENCES}

1. M. al-Bar and D. L. Johnson, Circular Braids, Arab Gulf J. Sci. Research 1:2 (1983). 
2. E. Artin, Theorie de zopfe, Abh. Math. Sem. Univ. Hamburg 4 (1925), 47-72.

3. E. A. Gorin and V. JA. LIN, Algebraic equations with continuous coefficients, and certain questions of the algebraic theory of braids, Math. USSR-Sb. 7 (1969), 569-596.

4. A. G. Kurosh, The Theory of Groups, II, 2nd ed. transl. and ed. K. A. Hirsch (New York: Chelsea, 1960).

5. A. K. NAPTHINE, On the Artin braid group and some of its analogues (Ph.D. Thesis, University of Nottingham, 1983).

6. R. J. WILSON, Introduction to Graph Theory, 2nd ed. (Longman, London, 1979).

Department of Pure Mathematics

UNIVERSITY OF SHEFFIELD

HiCKS BUILDING

SHEFFIELD S3 7RH 